\title{
Hirshfeld Atom Refinement of crystal structure and Hirshfeld surface analysis of five copper(II) fenamate complexes with $\mathbf{N}, \mathbf{N}$-diethylnicotinamide
}

\author{
Milan Piroš, Jozef Švorec, Jan Moncol \\ Slovak University of Technology, Bratislava, Slovak Republic; \\ jan.moncol@stuba.sk
}

Copper (II) complexes with NSAIDs are interesting as potential drugs with different biological activity such as potential anticancer and antioxidant activities (superoxide dismutase mimicking, radical scavenging and soybean lipoxygenase inhibition). ${ }^{1-3}$

A series of five copper(II) fenamate complexes with $N, N$-diethylnicotinamide ligand (den) of formula $\left[\mathrm{Cu}(\mathrm{nif})_{2}(\mathrm{den})_{2}\right](\mathrm{flu}=$ flufenamate) (1), $\left[\mathrm{Cu}(\mathrm{clo})_{2}(\mathrm{den})_{2}\right]\left(\mathrm{clo}=\right.$ clonixinate) $(\mathbf{2}),\left[\mathrm{Cu}(\mathrm{flu})_{2}(\mathrm{den})_{2}\left(\mathrm{H}_{2} \mathrm{O}\right)_{2}\right]($ flu $=$ flufenamate $)(\mathbf{3}),\left[\mathrm{Cu}(\mathrm{tol})_{2}(\mathrm{den})_{2}\left(\mathrm{H}_{2} \mathrm{O}\right)_{2}\right]($ tol $=$ tolfenamate) (4) and $\left[\mathrm{Cu}(\mathrm{mef})_{2}(\mathrm{den})_{2}\left(\mathrm{H}_{2} \mathrm{O}\right)_{2}\right](\mathrm{mef}=$ mefenamate) $(5)$ have been synthesized and structural characterized. The crystal structures of five complexes (1-5) were refined using the Hirshfeld Atom Refinement model (HAR) and Hirshfeld surface analysis have been also made.

[1] J.E. Weder, et al., Coord. Chem. Rev. 2002, 232, 95.

[2] C.N. Banti, S.K. Hadjikakou, Eur. J. Inorg. Chem. 2016, 3048.

[3] G. Psomas, Coord. Chem. Rev. 2020, 412, 213259.

Keywords: Aspheric refinement, copper(II) complexes, drugs, Hirshfeld surface analysis 